\title{
The use of gamma indices with medical imaging as quality assurance tool to validate the dose calculation algorithm in the modern practice of medical physics
}

\author{
Abdulhamid Chaikh ${ }^{1,2 *}$, Charline Desgranges ${ }^{1}$ and Jacques Balosso ${ }^{1,3}$ \\ ${ }^{1}$ Department of Radiation Oncology and Medical physics. University Hospital of Grenoble, France \\ ${ }^{2}$ France HADRON National Research Infrastructure, France \\ ${ }^{3}$ University Grenoble-Alpes - Grenoble, France
}

\begin{abstract}
Purpose: This study investigates the use of gamma indices $(\gamma)$ criteria and the digital imaging and communications in medicine (DICOM) images in order to compare dose distribution and readjust the prescription dose due to the change in dose calculation engine, to maintain the same clinical results when changing the dose calculation algorithm in a radiation oncology department.

Methods: Twelve treatment plans for lung cancer were analyzed. The dose calculation was performed using two different inhomogeneity correction methods using pencil beam convolution (PBC) turning on 1D and 3D density correction, respectively using the same beam arrangements.

This analysis was performed using $2 \mathrm{D}$ and $3 \mathrm{D} \gamma$ with a variety of dose-difference and distance-to-agreement: $1 \% / 1 \mathrm{~mm}, 2 \% / 2 \mathrm{~mm}, 3 \% / 3 \mathrm{~mm}, 3 \% / 1 \mathrm{~mm}$ and $3 \% / 2$ $\mathrm{mm}$. The average $\gamma$ values and percentages of pixels passing the $\gamma$ criteria were evaluated. For statistical analysis, Spearman's test was used to calculate the correlation coefficient "rho".

Results: The comparison between PBC-1D and PBC-3D showed that $95 \%$ of pixels have $\gamma \leq 1 \mathrm{with} 2 \% / 2 \mathrm{~mm}$ or $3 \% / 3 \mathrm{~mm}$. There is no impact of distance to agreement using global evaluation on $\gamma$ passing rates leading to the dose difference only related to turning on/off the 3D density correction instead of $1 \mathrm{D}$ density correction method. There was a weak correlation between $2 \mathrm{D}$ and $3 \mathrm{D} \gamma$ passing rates.

Conclusion: The 2D $\gamma$-maps and cumulative pixels- $\gamma$-histograms can be used to validate a new dose calculation algorithm and to make a medical decision to readjust the prescribed dose. The $3 \% / 3 \mathrm{~mm} \gamma$ criteria confirms that the modification of the prescription is not justified. However, a careful analysis should be taken for $\gamma$-maps concerning the organs at risks and of course; this method should be adjusted for different systems to be compared.
\end{abstract}

\section{Introduction}

In radiotherapy it is mandatory to deliver the prescribed dose to target volume with high accuracy. The dose prescribed by the radiation oncologist is prospectively assessed through different representations based on the calculation by specific algorithms implemented in the Treatment Planning System (TPS). The accuracy of representations varies according to the complexity of the anatomical structures and the performance of the calculations. In this context, tissues density heterogeneities are the main difficulties to overcome and have call for continuous progress in algorithms. The current algorithms are able to take into account the heterogeneity correction, i.e., pencil beam convolution $(\mathrm{PBC})$ with density correction methods, Anisotropic Analytical Algorithm (AAA) or Acuros XB implemented with Eclipse $^{\circledR}$ TPS and Collapsed Cone Convolution (CCC) implemented with Pinnacle ${ }^{\circledR}$ TPS [1-4]. Therefore, the change of dose calculation algorithm might be associated with the adjustment of dose prescription for clinical purpose, namely to obtain the same clinical results than using the former algorithm. Recently, Chaikh et al. 2014, showed that the implementation of a new dose calculation algorithm should be carefully validated into clinical practice, and they reported that the dose prescription might have to be readjusted. In the same time, the objective is to ensure that the dose delivered to the patient is, as close as possible, to the prescribed dose and reducing the dose burden to healthy tissues as much as possible [2]. In this study, we validate the $2 \mathrm{D}$ $\gamma$ indices as a quality assurance (QA) tool to safely implement modern dose calculation algorithms and the choice of the appropriate $\gamma$ criteria to use for this. To validate this method in its principle, the PBC turning on $1 \mathrm{D}$ and $3 \mathrm{D}$ density correction methods were used.

\section{Material and methods}

\section{Treatment planning and dose calculation}

This study is based on twelve treatment plans for lung cancer. The patients were irradiated using $3 \mathrm{D}$ conformal radiation therapy. A computed tomography (CT-scan) was carried out for each patient. Then, the images were loaded into the Eclipse ${ }^{\circledR}$ TPS (Version 8.1; Varian Medical Systems). The dose calculation was performed using

Correspondence to: Abdulhamid Chaikh, Department of Radiation Oncology and Medical physics, University Hospital of Grenoble, France, Tel: +33 62573 78 99, Fax: +33 476765629; E-mail: abdulhamedc@yahoo.com

Key words: $P B C$ with density correction methods, gamma analysis, radiotherapy Received: May 07, 2016; Accepted: June 27, 2016; Published: June 30, 2016 
Chaikh A (2016) The use of gamma indices with medical imaging as quality assurance tool to validate the dose calculation algorithm in the modern practice of medical physics

two different inhomogeneity correction methods integrated in PBC. The PBC model takes into account density information from CT-scan either in one-dimensional (PBC-1D), such as modified batho method, or the full three-dimensions (PBC-3D) of along a ray path from the source to the point of interest, such as equivalent tissue air ratio method [5-8]. Both methods use an empirical inhomogeneity correction factor to account for both primary beam attenuation and scatter changes.

\section{Gamma analysis}

To make an overall comparison, 2D and 3D indices were used. This tool combines two criteria including the dose-difference in percentage (\%) and the Distance to Agreement (DTA) in millimeters (mm). An ellipse is used to determine the acceptance region, $\leq 1$ represents fulfillment of the criteria [9,10]. For 2D analysis, the digital imaging and communications in medicine (DICOM) images including dose distribution, planer dose, from PBC-1D and PBC-3D for each patient were exported from TPS to RIT-113 ${ }^{\circledR}$ (Dosimetry System Version 5.2). The dimensions were $20 \times 20 \mathrm{~cm}^{2}$ with the resolution was $0.39 \mathrm{~mm}$. The $2 \mathrm{D}$ per treatment plan was calculated by considering all pixels for a specific patient using transverse, sagittal and coronal planes. The results were displayed using a $2 \mathrm{D}$-maps and cumulative Pixels- -Histogram (P $\mathrm{H})$. The -maps show the pixels with $\gamma>1$ that were out of tolerance, indicating over/under estimating dose. In order to discriminate an over from an under estimated dose, a color-code was associated with dose difference. For over estimating dose, the dose-difference was presented in red coloring, indicating DPBC-1D $>$ DPB-3D. For underestimating dose, the dose-difference was presented in blue coloring, indicating DPBC-1D < DPBC-3D. Hence, the red and blue zones in 2D -maps show respectively over and under estimated doses, resulting from $\mathrm{PBC}$ $1 \mathrm{D}$ compared with PBC-3D. The superposition of the 2D -map with the CT-scan introduced the anatomic information showing in color where the dose differences are. We could then see the healthy tissues located around the target volumes. The $\mathrm{P} \mathrm{H}$ indicates the fraction of pixels with a -indices equal or lower than a specific value. In $3 \mathrm{D}$ analysis, the DICOM images including dose distribution, from both PBC methods, including target volumes and organ at risks (OARs) of each patient were exported from TPS to Slicer RT [11]. To generate $2 \mathrm{D}$ and $3 \mathrm{D}$ evaluations, the pixels or voxels with lower doses $<10 \%$ were disregarded for the quantitative analysis. Five sets of criteria were used: $1 \% / 1 \mathrm{~mm}, 2 \% / 2 \mathrm{~mm}, 3 \% / 3 \mathrm{~mm}, 3 \% / 1 \mathrm{~mm}$ and $3 \% / 2 \mathrm{~mm}$. We considered that dose distributions from PBC were similar, if $95 \%$ of pixels or voxels passing $\gamma$ criteria with $\leq 1$.

\section{Statistical analysis}

Spearman's rank test was used to calculate the correlation coefficient "rho" [12]. In addition, the Wilcoxon signed rank test was used to evaluate whether the differences between the $2 \mathrm{D}$ and $3 \mathrm{D}$ data was statistically significant and calculate the $p$-value, $p<0.05$ was considered as a significance difference.

\section{Results}

\section{D gamma analyses}

Table 1 shows the results of $\gamma$ for axial planes including $\gamma$-max, $\gamma$-mean with standard deviation (SD) and the pixels having $\gamma \leq 1$. It can be seen in table 1 PBC methods, did not yield much difference using $2 \% / 2 \mathrm{~mm}$ or $3 \% / 3 \mathrm{~mm}$. Figure 1 shows a sample of a $2 \mathrm{D} \gamma$-maps in the axial, coronal and sagittal views. The $2 \mathrm{D} \gamma$-maps were calculated

Table 1. Results of 2D gamma for transverse planes including $\gamma$-max, $\gamma$-mean with SD and the percentage of pixels having $\gamma \leq 1$, by varying dose-difference and DTA criteria.

\begin{tabular}{|c|c|c|c|}
\hline Acceptance criteria & Average 2D $\boldsymbol{\gamma}$-max & $\begin{array}{c}\text { Average 2D } \boldsymbol{\gamma} \text {-mean } \\
\pm \mathbf{S D}\end{array}$ & $\begin{array}{c}\text { Average 2D percentage of } \\
\text { pixels passing } \boldsymbol{\gamma}\end{array}$ \\
\hline $1 \% / 1 \mathrm{~mm}$ & 4.6 & $0.5 \pm 0.1$ & 86.2 \\
\hline $2 \% / 2 \mathrm{~mm}$ & 2.0 & $0.2 \pm 0.2$ & 98.6 \\
\hline $3 \% / 3 \mathrm{~mm}$ & 1.3 & $0.1 \pm 0.1$ & 99.9 \\
\hline $3 \% / 2 \mathrm{~mm}$ & 1.4 & $0.2 \pm 0.2$ & 99.9 \\
\hline $3 \% / 1 \mathrm{~mm}$ & 1.4 & $0.2 \pm 0.2$ & 99.9 \\
\hline
\end{tabular}

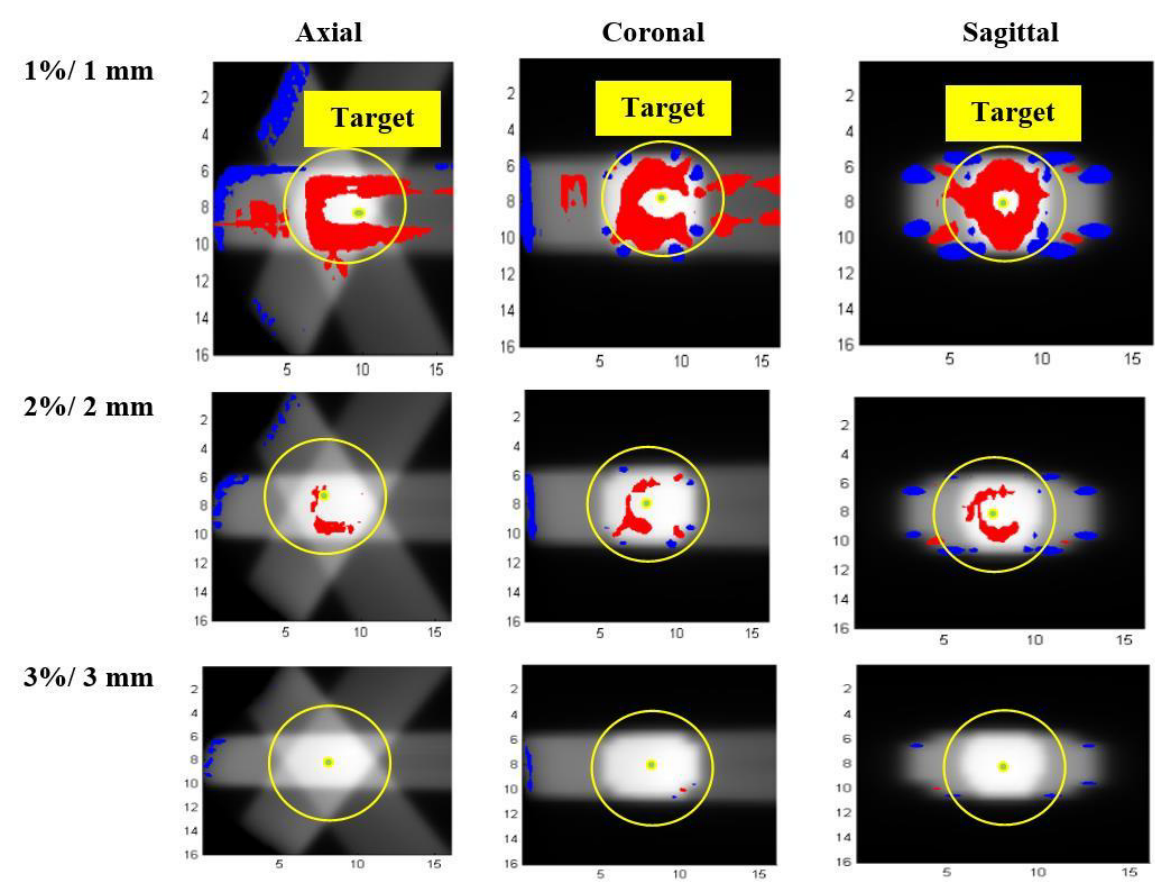

Figure 1. 2D- $\gamma$ maps plotted on the axial, coronal and sagittal views for comparing PBC-1D with PBC-3D. The red and blue coloring indicate that $\gamma>1$ and identifying respectively over/ under estimating dose. The yellow circles indicate the targets contours around the isocentre point in all planes. 
Chaikh A (2016) The use of gamma indices with medical imaging as quality assurance tool to validate the dose calculation algorithm in the modern practice of medical physics

using DICOM images including tumor and organs at risks for lung cancer. The red and blue coloring indicate that $\gamma>1$ and identifying over estimating dose (DPBC-1D $>$ DPBC-3D) or underestimating dose (DPBC-1D $<$ DPBC-3D), respectively.

Figure 2 shows the $\mathrm{P} \gamma \mathrm{H}$ from $1 \% / 1 \mathrm{~mm}, 2 \% / 2 \mathrm{~mm}$ and $3 \% / 3$ $\mathrm{mm}$ criteria's. It can be seen that $100 \%$ and $99.9 \%$ of pixels have $\gamma \leq 1$ using $2 \% / 2 \mathrm{~mm}$ and $3 \% / 3 \mathrm{~mm}$, respectively. Nevertheless, using $1 \% / 1$ mm, only $94 \%$ of pixels with $\gamma \leq 1$ was observed. This mean that the tolerance $95 \%$ of pixels with $\gamma \leq 1$ was satisfied using both $\gamma$ criteria, $2 \% / 2 \mathrm{~mm}$ and $3 \% / 3 \mathrm{~mm}$.

\section{$D$ gamma analyses}

Figure 3 shows the results of $3 \mathrm{D} \gamma$ passing rates indicating the voxels with $\gamma \leq 1$. The average percentage of voxels passing $\gamma$ were $80.8 \%$, $94.8 \%$ and $99.2 \%$ for $1 \% / 1 \mathrm{~mm}, 2 \% / 2 \mathrm{~mm}$ and $3 \% / 3 \mathrm{~mm}$, respectively; and $98.5 \%$ for $3 \% / 2 \mathrm{~mm}$ and $3 \% / 1 \mathrm{~mm}$.

\section{Correlation between $2 \mathrm{D}$ and $3 \mathrm{D}$ gamma passing rates}

The data resulting from $2 \mathrm{D}$ and $3 \mathrm{D} \gamma$ showed a weak correlation for $1 \% / 1 \mathrm{~mm}, 3 \% / 3 \mathrm{~mm}, 3 \% / 2 \mathrm{~mm}$ and $3 \% / 1 \mathrm{~mm}$ with "rho" $<0.5$. However, a good correlation was observed for $2 \% / 2 \mathrm{~mm}$. The difference between $2 \mathrm{D}$ and $3 \mathrm{D} \gamma$ for percentage of pixels/voxels passing $\gamma$ was statistically significant, $\mathrm{p}<0.01$. Figure 4 shows correlation coefficient, for $2 \mathrm{D}$ and $3 \mathrm{D} \gamma$ passing rates generated for all $\gamma$ criteria.

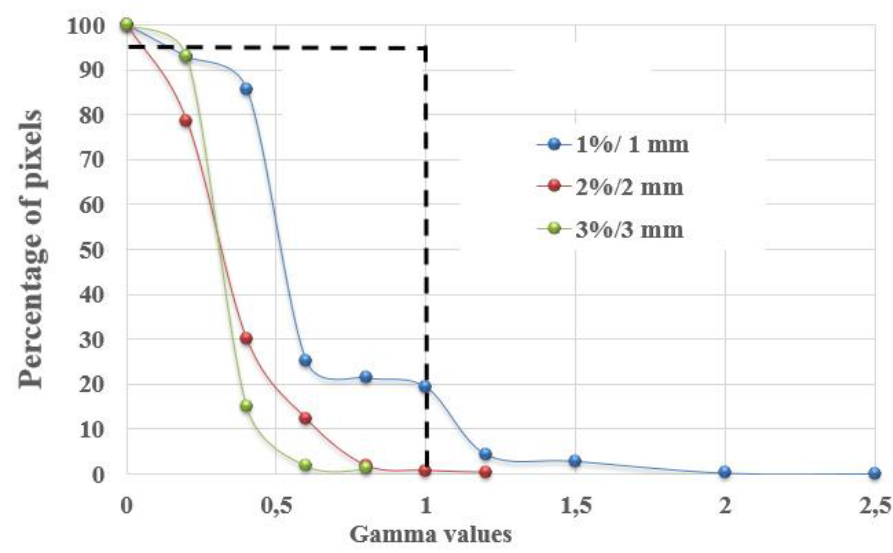

Figure 2. Cumulative $\mathrm{P} \gamma \mathrm{H}$, obtained from comparison of $\mathrm{PBC}-1 \mathrm{D}$ and $\mathrm{PBC}-3 \mathrm{D}$ for one patient using three $\gamma$ criteria's. We note that the $\gamma$ passing rates, $95 \%$ of pixels with $\gamma \leq 1$, was satisfied using $2 \% / 2 \mathrm{~mm}$ or $3 \% / 3 \mathrm{~mm}$.

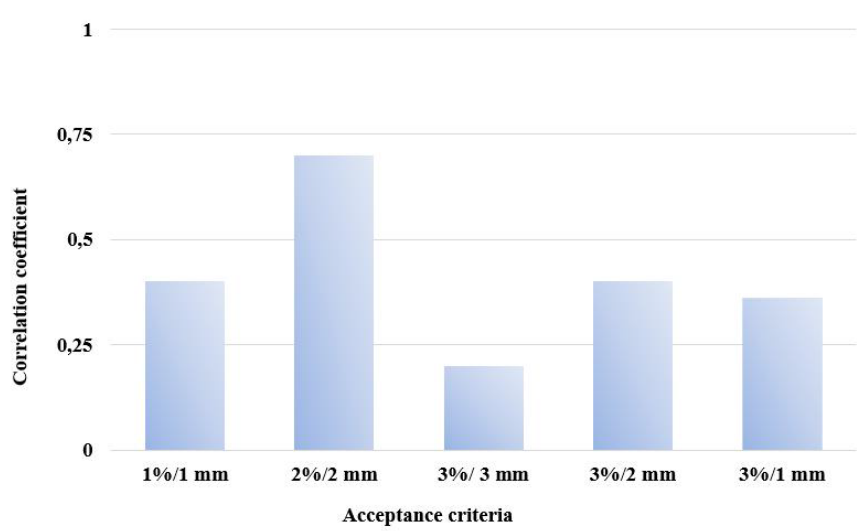

Figure 3. The results of 3D $\gamma$ passing rates indicating the percentage of voxels with $\gamma \leq 1$, by varying dose-difference and DTA criteria.

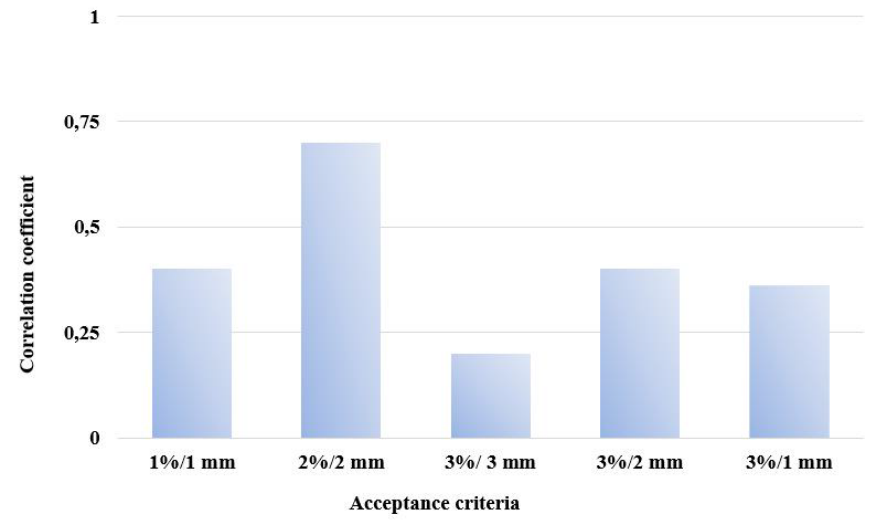

Figure 4. Correlation coefficient from 2D and 3D gamma passing rates using all gamma criteria for all patients.

\section{Discussion}

Relevant gamma indices criteria to readjust the prescribed dose

In this study, we compared two algorithms based on PBC-1D and PBC-3D density correction methods using exactly the same beam arrangements. For $\gamma$ analysis, five criteria were used by varying DTA and dose-difference. Firstly, there is no impact on -value by varying DTA and fixing dose-difference, since the comparison of both algorithms is carried out using exactly the same beam geometry, as shown in Table 1. Therefore, the passing rates reflect only the dose difference, and not any geometrical alteration, resulting from algorithm change. Concerning dose differences, the CL with $2 \mathrm{D} \gamma$ was $<1 \%$ using $3 \% / 3$ $\mathrm{mm}$. Consequently, to make a decision regarding any dose prescription alteration when moving from PBC-1D to PBC-3D, it seems possible to compare dose calculation algorithms, for the same patient and the same arrangement, with the more sensitive criteria $2 \%$ than $3 \%$. One can discard any modification of prescription if the usual qualitative evaluation of the cumulative $\mathrm{P} \mathrm{H}$ is above the threshold of $95 \%$ of pixels having $\leq 1$. In this, it would be allowed to conclude an acceptable dose calculation similarity between both algorithms.

\section{Advantage and limit of this study}

The objective of analysis is to validate with safety a fast and convenient method to test the need of adjustment of dose prescription when the modern dose calculation algorithm is implemented.

The $\gamma$ method proposed in this study present two advantages compared to classical tools to validate a treatment plans.

Firstly, it is a familiar and fast QA tool for medical physicists, since the principle is similar to that used to validate a treatment plan in radiotherapy. In general, there are two conditions to validate treatment plan, regarding to target and OARs, based on spatial distribution with isodose curves and dosimetric parameters deriving from cumulative dose volume histogram (DVH). The isodose curve of $95 \%$ should cover the target and in the other hand, the delivered dose to target should be more than $95 \%$ and less than $107 \%$ of the prescribed dose. For OARs, the dose constraint must be respected (for example: V20 Gy $<30 \%$ and $\mathrm{V} 30 \mathrm{~Gy}<20 \%$ for lung tolerance). However, the information related to DVH is not sufficient to alert medical physicists and radiotherapists about the risk of under/over estimating dose, since it does not provide any information about the spatial dose distribution. It reports only a numerical value for a possible hot spot for target or OARs with no 

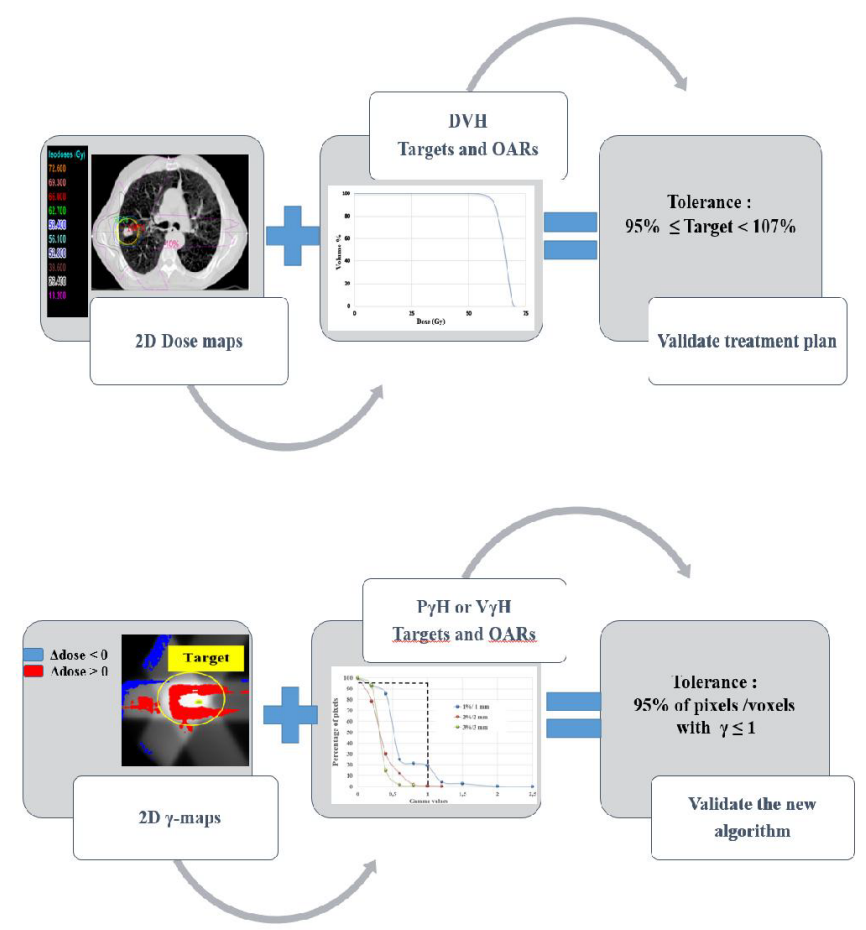

Figure 5. Comparison between $2 \mathrm{D}$ dose maps with DVH tools to validate a treatment plan in radiotherapy and the $2 \mathrm{D}$ gamma with $\mathrm{P} \gamma \mathrm{H}$ tools to validate and implement a dose calculation algorithm.

localization of this point.

Thus, the second advantage of $\gamma$ analysis is to show a spatial representation of the dose differences, the $\gamma$-maps, and a quantitative comparison of the differences using $\mathrm{P} \gamma \mathrm{H}$. The $\gamma$-maps is similar to a dose distribution map. The $\gamma$-map is showing the areas, either in the target or in the OARs, that does not respect the threshold of acceptable difference. In this case, the volumes having $\gamma>1$ are represented in color code showing the over/under estimating dose. For example, it can be seen an overestimated dose located in small areas of healthy lung presenting an OARs using $1 \% / 1 \mathrm{~mm}$ criteria. This is an alert for the physicist and the radiation oncologist that the $\gamma$ passing rates for OARs is not satisfied. The $\mathrm{P} \gamma \mathrm{H}$ is similar to cumulative $\mathrm{DVH}$, in this case, the $95 \%$ of pixels or voxels should have $\gamma \leq 1$. Figure 5 illustrates a comparison between $2 \mathrm{D}$ dose maps with DVH tools to validate a treatment plan in radiotherapy and the $2 \mathrm{D} \gamma$ with $\mathrm{P} \gamma \mathrm{H}$ tools to validate and implement a dose calculation algorithm. However, this study has some limits. The dose calculation algorithm is based on density correction methods integrated with PBC. Currently, modern algorithms are available for dose calculations. This method can, in principle, be used to explore the correlation between former and newer algorithms of any types. However, the appropriate level of analysis, as here $2 \% / 2 \mathrm{~mm}$ must be assessed and if necessary adapted to other conditions of use.

\section{Conclusion}

We validated a fast QA method to evaluate the dose-difference and make possible to readjust the prescribed dose when the modern dose calculation algorithm should be integrated. This method is based on $2 \mathrm{D}$ and $3 \mathrm{D} \gamma$ evaluation including $\gamma$-maps and $\mathrm{P} \gamma \mathrm{H}$. For the specific comparison between PBC-1D and PBC-3D, used a study model, the $\gamma$ criteria $2 \% / 2 \mathrm{~mm}$ proved to be sufficiently correlated to doses differences to be used as a surrogate of the direct dose comparison, which is long and difficult. It can be considered that no significant differences are to be considered if $95 \%$ of pixels have $\gamma \leq 1$, i.e., no dose modification to consider for the prescribed dose. However, a careful analysis should be taken for $\gamma$-maps concerning the organs at risks and of course; this method should be adjusted for different systems to be compared.

\section{Conflict of interest}

The authors declare that they have no conflicts of interest. The authors alone are responsible for the content and writing of the paper.

\section{References}

1. Knoos T, Wieslander E, Cozzi L, Brink C, Fogliata A, et al. (2006) Comparison of dose calculation algorithms for treatment planning in external photon beam therapy for clinical situations. Phys Med Biol 51: 5785-5807. [Crossref]

2. Chaikh A, Balosso J (2014) Should the dose prescription be readjusted when using tissues density corrections algorithms for radiation oncology J Case Rep Onc Ther 1: 01018 .

3. Rana S (2014) Clinical dosimetric impact of Acuros XB and analytical anisotropic algorithm (AAA) on real lung cancer treatment plans: review. Int J Cancer Ther Oncol 2: 02019 .

4. Ojala J (2014) The accuracy of the Acuros XB algorithm in external beam radiotherapy - a comprehensive review. Int J Cancer Ther Oncol 2: 020417.

5. Task Group No. 65, the Radiation Therapy Committee of the American Association of Physicists in Medicine. Tissue inhomogeneity corrections for MV photon beams. Madison, WI: Medical Physics Publishing, 2004.

6. Ahnesjö A, Aspradakis MM (1999) Dose calculations for external photon beams in radiotherapy. Phys Med Biol 44: R99-155. [Crossref]

7. BATHO HF (1964) Lung Corrections In Cobalt 60 Beam Therapy. J Can Assoc Radiol 15: 79-83. [Crossref]

8. El-Khatib E, Battista JJ (1984) Improved lung dose calculation using tissue-maximum ratios in the Batho correction. Med Phys 11: 279-286. [Crossref]

9. Spezi E, Lewis DG (2006) Gamma histograms for radiotherapy plan evaluation. Radiother Oncol 79: 224-230. [Crossref]

10. Chaikh A, Giraud JY, Balosso J (2014) A 3D quantitative evaluation for assessing the changes of treatment planning system and irradiation techniques in radiotherapy. Int $J$ Cancer Ther Oncol 2: 02033.

11. Pinter C, Lasso A, Wang A, Jaffray D, Fichtinger G (2012) SlicerRT: radiation therapy research toolkit for 3D Slicer. Med Phys 39: 6332-6338. [Crossref]

12. Chaikh A, Giraud JY, Perrin E, Bresciani JP, Balosso J (2014) The choice of statistical methods for comparisons of dosimetric data in radiotherapy. Radiat Oncol 9: 205. [Crossref]

Copyright: (C)2016 Chaikh A. This is an open-access article distributed under the terms of the Creative Commons Attribution License, which permits unrestricted use, distribution, and reproduction in any medium, provided the original author and source are credited. 